\title{
Using a wireless consumer accelerometer to measure tibial acceleration during running: agreement with a skin-mounted sensor
}

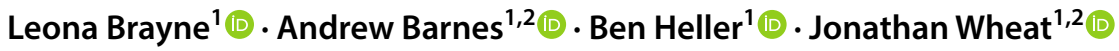

Published online: 15 May 2018

(c) The Author(s) 2018

\begin{abstract}
Real-time monitoring and feedback of tibial acceleration using wireless skin mounted sensors may reduce the risk of tibial stress fractures in runners. The purpose of this study was to assess the agreement between a wireless accelerometer and a gold standard reference accelerometer, both skin mounted, in measuring peak axial tibial acceleration when treadmill running at a range of speeds. A research grade accelerometer was mounted to a wireless accelerometer and attached to the tibia. Peak positive tibial accelerations of 13 participants were compared at 2.5, 3.5 and $4.5 \mathrm{~m} \mathrm{~s}^{-1}$. Intraclass correlation coefficients demonstrated good agreement, with limits of agreement showing accuracy to within 1.2-1.65 g. The wireless accelerometer has scope to be used as a tool to measure peak tibial accelerations during running for the purpose of real-time feedback in gait training systems.
\end{abstract}

Keywords Tibial shock $\cdot$ Real-time feedback $\cdot$ Reliability $\cdot$ Gait retraining

\section{Introduction}

Both prospective [1] and retrospective [2] studies suggest that large peak tibial accelerations are a strong risk factor for tibial stress injuries in runners. Bone mounted accelerometers are perhaps the true 'gold standard' method for measuring tibial acceleration [3]. However, ethical issues mean that skin-mounted devices are more routinely used in running analysis to identify those at an elevated risk of injury $[4,5]$. With appropriate location and application procedures, differences between skin and bone mounted accelerometers can be minimised [6]. As such, skin-mounted devices are considered suitable for measuring tibial accelerations [7]. However, they are relatively expensive and their use is often confined to a laboratory-limiting their application within injury risk assessment, rehabilitation and gait retraining.

Leona Brayne

1.brayne@shu.ac.uk

1 Centre for Sports Engineering Research, Sheffield Hallam University, Collegiate Hall, Collegiate Crescent, Sheffield, England S10 2BP, UK

2 Academy of Sport and Physical Activity, Sheffield Hallam University, Collegiate Hall, Collegiate Crescent, Sheffield, England S10 2BP, UK
Recently, consumer sensor technologies incorporating accelerometers have become widely available. Compared to research grade accelerometers, consumer sensors are low cost (cost: $<\$ 100$ ), easy to use, wireless, and waterproof. As such, these devices offer the potential for measurement and feedback of tibial acceleration outside of the laboratory, which could lead to new paradigms for use in injury assessment, rehabilitation and retraining. However, despite great potential, using consumer devices to measure tibial acceleration has not been explored. Therefore, the aim of this study was to examine the agreement between a skin mounted, consumer wireless accelerometer and a skin mounted, wired, research-grade accelerometer (gold standard) in measuring peak tibial acceleration.

\section{Materials and methods}

Following ethical approval (Sheffield Hallam University Ethics Board), 13 male rearfoot strikers were recruited for the study ( $30 \pm 7$ years; height $1.78 \pm 0.08 \mathrm{~m}$; mass $77 \pm 10 \mathrm{~kg})$. Only rearfoot strikers were recruited to ensure participants displayed similar loading characteristics [4]. All participants were running at least $16 \mathrm{~km}$ per week and free from any lower limb musculoskeletal injury at the time of testing. Two accelerometers were used to measure tibial acceleration: (1) 
A consumer wireless accelerometer (RunScribe version 1, Scribe Labs, California, USA) containing an MPU-9150 inertial measurement unit (Invensense, California comprising a tri-axial accelerometer, magnetometer and rate gyroscope) encased in a housing (total mass, $9.55 \mathrm{~g}$ ) (Fig. 1a) and (2) a research grade uniaxial piezoresistive accelerometer (model 352C22, PCB Piezotronics, Stevenage, UK), considered a gold standard (reference sensor), and used in previous studies to measure tibial acceleration during running-with a between days reliability of 0.87 ( $\left.\mathrm{ICC}_{2,1}\right)$ [5] (Fig. 1b). The reference sensor was attached to a small piece of thermoplastic (total mass, $1.65 \mathrm{~g}$ ) (Fig. 1b); connected via a wire to a PCB signal conditioner mounted remotely (model 480E09; gain $=10$ ). Although the sensor could not be attached to the bone and was still skin mounted, acceptable accuracy has been reported for skin mounted sensors with a mass of less than $3 \mathrm{~g}$ [7]. This supports the choice of the reference sensor in offering the most accurate means of measuring tibial acceleration in vitro. Both sensors were sampled at $1000 \mathrm{~Hz}$.

The reference accelerometer was mounted using doublesided tape to the largest surface of the consumer accelerometer with the sensitive axes of both aligned visually (Fig. 2). The two adjoined sensors were attached to the distal anteromedial aspect of the tibia, $5 \mathrm{~cm}$ above the medial malleolus [4] using double-sided tape, ensuring that the sensitive axes of the sensors were aligned with the long axis of the tibia. This site was chosen due to the thin layer of soft tissue overlying the bone, thus reducing the effect of soft tissue oscillations generated during impact [8]. Tension was applied to the skin at the attachment site to help minimise soft tissue motion [6], and the sensors tightly over wrapped with elastic bandage about the circumference of the shank. To ensure consistency across participants, the same investigator applied the sensors on each occasion.

Following a warm up, participants ran at three different speeds on a treadmill in a randomised order categorised as low: $\left(2.5 \mathrm{~m} \mathrm{~s}^{-1}\right)$, medium: $\left(3.5 \mathrm{~m} \mathrm{~s}^{-1}\right)$, and high: $\left(4.5 \mathrm{~m} \mathrm{~s}^{-1}\right)$. Participants were allowed to rest between trials to avoid fatigue. Each trial involved the participant running at the target speed for a total of $40 \mathrm{~s}$, with $10 \mathrm{~s}$ to regulate running gait and then a $30 \mathrm{~s}$ data collection period. In a further set

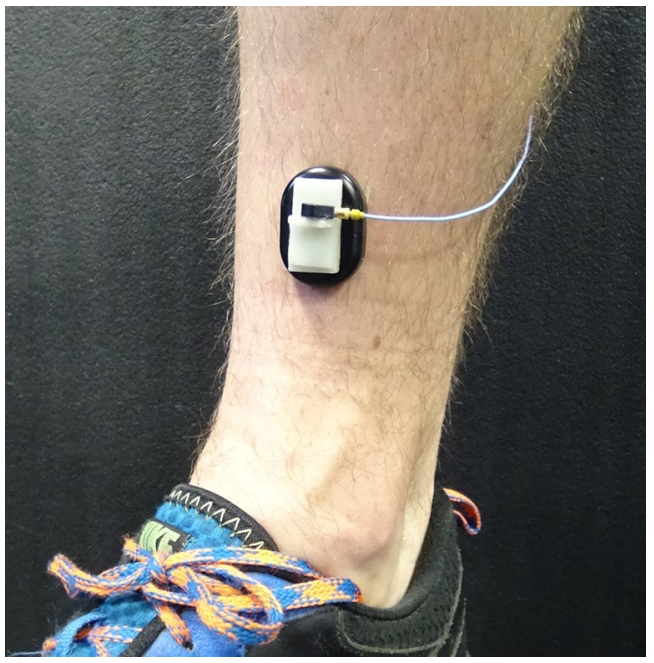

Fig. 2 Alignment and attachment of both sensors on the tibia when mounted concurrently (prior to overwrapping)

of trials, the reference sensor was mounted independently at the same location to assess the agreement of the sensor when attached directly to the skin and when mounted on the consumer accelerometer. All trials were completed in a single session and participants wore their own running shoes.

All data from both sensors were bandpass filtered between 2 and $75 \mathrm{~Hz}$ with a 2nd order Butterworth filter and converted to units of $\mathrm{g}$ using custom Matlab software (Mathworks, R2014a). Residual analysis of the data of ten participants across velocities determined the filter cut off choice [6]. The sensors were synchronised by way of the participant stamping their foot before starting a run so that the same series of foot strikes were analysed for the data from each sensor. Peak positive tibial accelerations-defined as the maximum value during stance-were calculated for each foot strike using both sensors to allow direct peak to peak comparisons across the $30 \mathrm{~s}$ run.

Intraclass correlation coefficients $\left(\mathrm{ICC}_{2,1}\right)$ and $95 \%$ limits of agreement (LOA) were used to assess sensor agreement. An ICC value $>0.75$ was considered good, whilst $0.4-0.75$ was considered moderate [9]. Narrower confidence intervals
Fig. 1 a Consumer accelerometer and $\mathbf{b}$ reference accelerometer. British one pence coin also shown for scale a

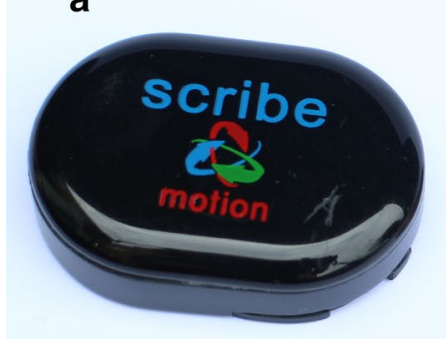

b

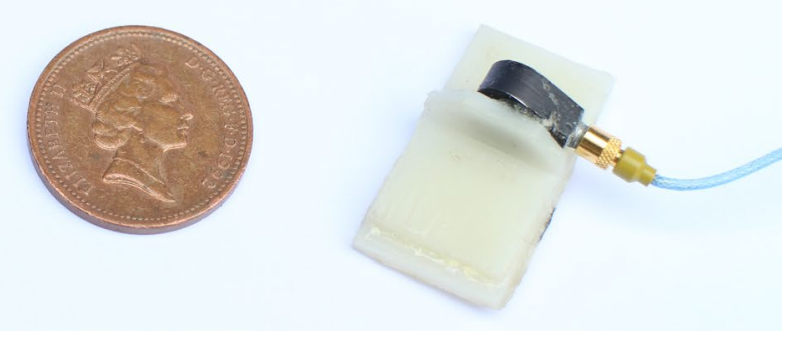


(CI) of the LOA were considered an indication of the agreement between sensors with an acceptable level of agreement determined specifically for the application of developing real time feedback systems for runners. As the data were normally distributed, a paired samples $t$ test was used to assess agreement of peak acceleration using the gold standard accelerometer when attached to the consumer accelerometer and when mounted independently $(P<0.05)$.

\section{Results}

There were no significant differences between peak tibial accelerations measured with the reference accelerometer attached directly to the skin and when it was mounted on the consumer accelerometer at each of the run speeds: $2.5 \mathrm{~m} \mathrm{~s}^{-1}, p=0.29 ; 3.5 \mathrm{~m} \mathrm{~s}^{-1}, p=0.76 ; 4.5 \mathrm{~m} \mathrm{~s}^{-1}$, $p=0.13$.

The comparison of mean peak acceleration between sensors using $\mathrm{ICC}_{(2,1)}$ indicated good agreement of the consumer accelerometer with the reference accelerometer at each run speed: $2.5 \mathrm{~m} \mathrm{~s}^{-1}(\mathrm{ICC}=0.92), 3.5 \mathrm{~m} \mathrm{~s}^{-1}$ $(\mathrm{ICC}=0.90), 4.5 \mathrm{~m} \mathrm{~s}^{-1}(\mathrm{ICC}=0.89)$ (Table 1$)$. An example portion of both sensor acceleration traces for one participant can be seen in Fig. 3. Mean differences in peak tibial acceleration for the concurrent data collection ranged from 0.23 to $0.36 \mathrm{~g}$ across running speeds (Table 1 ). Acceptable LOA were observed between the sensors, but some random differences were noted with $95 \%$ confidence interval ranging from 1.20 to $1.65 \mathrm{~g}$ across running speeds (Fig. 4).

Table 1 Summary of results for peak tibial acceleration during running: wireless accelerometer compared to the reference accelerometer

\begin{tabular}{|c|c|c|c|c|c|c|c|c|c|}
\hline \multirow[t]{2}{*}{ Sensor } & \multirow{2}{*}{$\begin{array}{l}\text { Run speed } \\
\left(\mathrm{m} \mathrm{s}^{-1}\right)\end{array}$} & \multirow{2}{*}{$\begin{array}{l}\text { Mean accelera- } \\
\text { tion } \pm \mathrm{SD}(\mathrm{g})\end{array}$} & \multicolumn{3}{|l|}{ ICC } & \multicolumn{4}{|l|}{ LOA } \\
\hline & & & Single measure & Lower bound & Upper bound & $\begin{array}{l}\text { Mean dif- } \\
\text { ference }(\mathrm{g})\end{array}$ & $95 \% \mathrm{CI}$ & Lower CI & Upper CI \\
\hline Reference & 2.5 & $4.04 \pm 1.55$ & 0.92 & 0.76 & 0.97 & 0.24 & 1.20 & -0.96 & 1.44 \\
\hline Consumer & & $4.28 \pm 1.57$ & & & & & & & \\
\hline Reference & 3.5 & $5.92 \pm 1.83$ & 0.90 & 0.71 & 0.97 & 0.23 & 1.65 & -1.42 & 1.88 \\
\hline Consumer & & $6.15 \pm 1.92$ & & & & & & & \\
\hline Reference & 4.5 & $7.88 \pm 1.87$ & 0.89 & 0.66 & 0.97 & 0.36 & 1.57 & -1.21 & 1.94 \\
\hline Consumer & & $8.24 \pm 1.73$ & & & & & & & \\
\hline
\end{tabular}

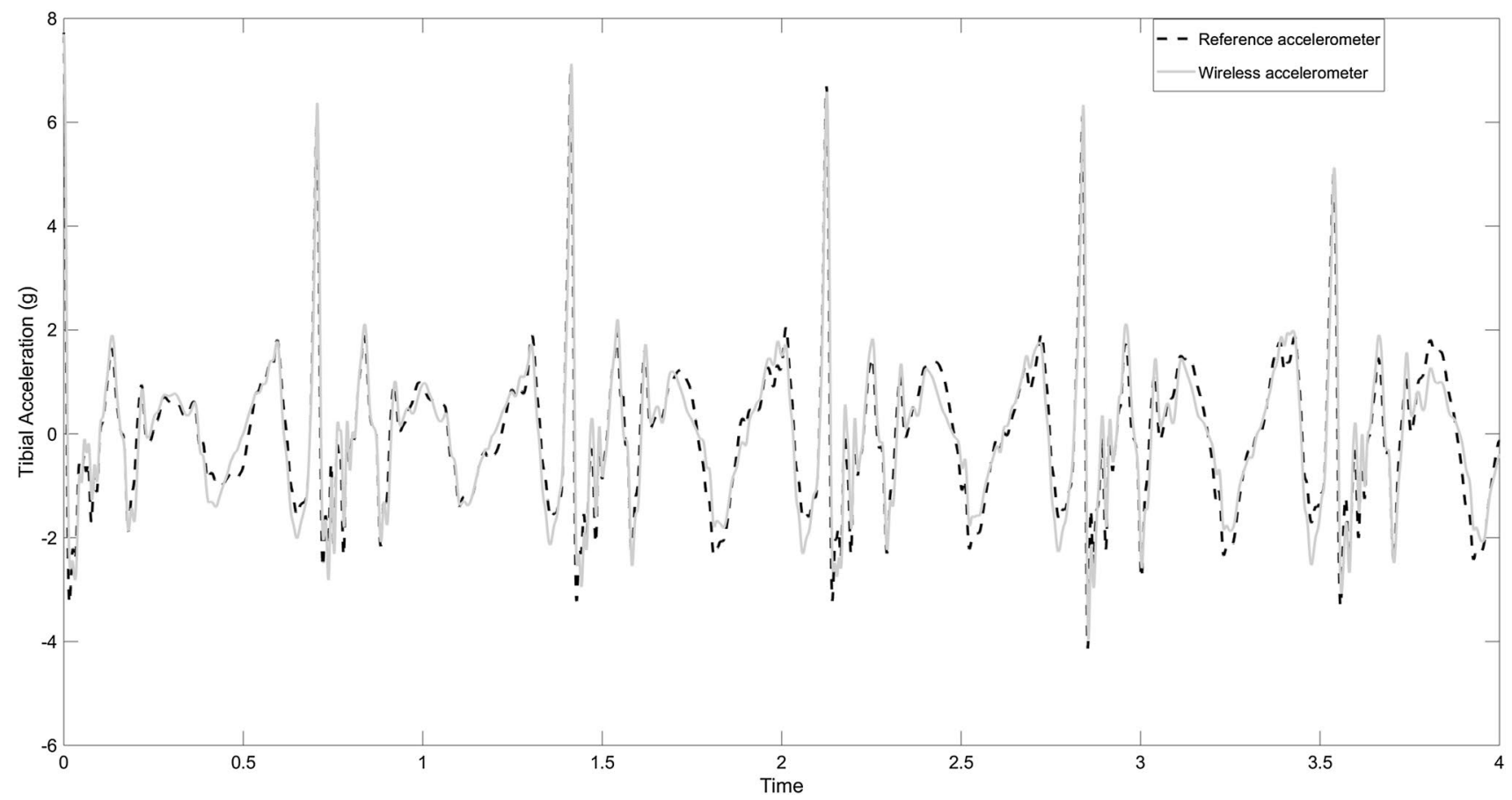

Fig. 3 Peak tibial acceleration traces for a typical participant running at $3.5 \mathrm{~m} \mathrm{~s}^{-1}$ as measured from both the reference and consumer accelerometer 

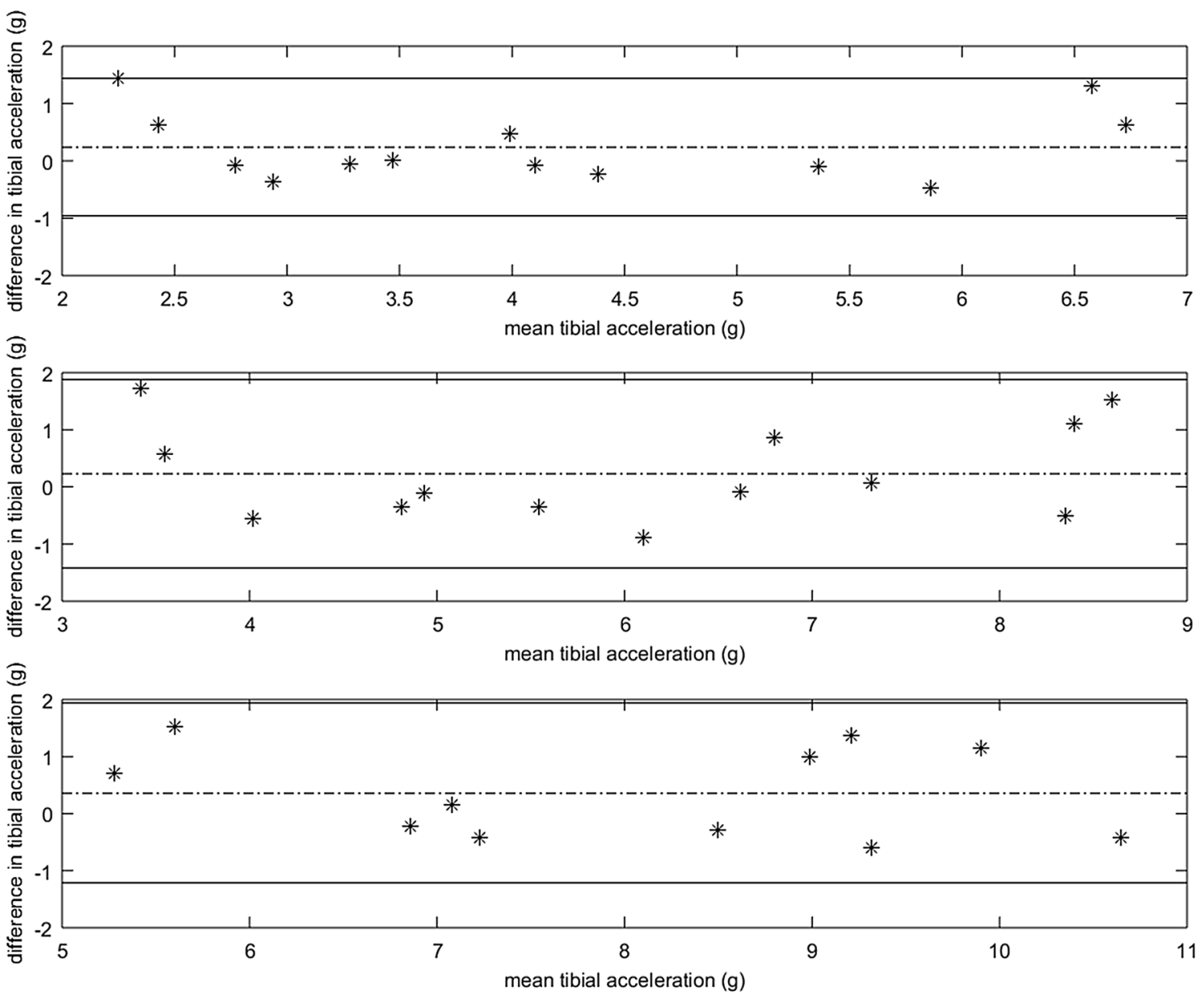

Fig. 4 Limits of agreement for peak tibial acceleration for running at all three speeds: consumer accelerometer compared to the reference accelerometer (dotted line represents systematic difference, solid line represents upper and lower bounds of limits of agreement)

\section{Discussion}

This study examined the agreement of a skin-mounted consumer accelerometer and a skin-mounted reference (gold standard) accelerometer in measuring peak tibial acceleration during running; to determine its suitability as a wearable sensor for use outside of the lab in, for example, a real-time feedback gait retraining system. Mean peak tibial acceleration values were similar to those reported in previous research $[4,5]$ with greater accelerations seen with increased run speed [10]. Results suggest good agreement between sensors across running speeds, with small mean differences in peak tibial acceleration and acceptable confidence intervals. With previous research reporting up to $50 \%$ reductions in peak tibial acceleration following real-time feedback interventions $[11,12]$ the LOA were deemed acceptable for this specific application. Therefore, our results indicate that the consumer accelerometer is capable of accurately measuring peak tibial acceleration at a range of running speeds and support the use of the sensor in representative settings, outside of the laboratory in, for example, bio feedback related gait retraining systems.

This study had some limitations which should be considered when interpreting the results. Previous research has found skin mounted sensors to significantly overestimate accelerations compared to bone mounted sensors, with differences of up to $2.1 \mathrm{~g}$ [3]. Steps were taken to minimise this error with the careful choice of sensor location and attention to application procedures which have been shown to impact significantly on measured values [8]. Mounting the reference accelerometer onto the consumer accelerometer resulted in a relatively large combined mass, which could have affected the degree to which accelerations measured using the skin-mounted sensors reflected the acceleration of the tibia. However, no significant differences were found between the peak accelerations recorded by the reference accelerometer mounted independently and with the two sensors mounted together. In addition, although the sensor axes were visually aligned, minor misalignment of the axes of both sensors may have caused cross talk [10]. 


\section{Conclusions}

The results of this study suggest that the consumer accelerometer accurately measures peak tibial accelerations when compared to a research grade skin mounted accelerometer, at a range of speeds. The consumer accelerometer is low cost, easy to use, wireless, and waterproof; it, therefore, represents a suitable tool for providing real time feedback to runners in the field. Real-time feedback of peak tibial acceleration during running using consumer accelerometers will aid sports scientists and coaches in monitoring and reducing peak tibial accelerations experienced by runners. Further research should seek to integrate this sensor into real-time feedback systems to be worn by runners in the field and investigate the efficacy of interventions based on these systems.

\section{Compliance with ethical standards}

Conflict of interest The authors declare no conflict of interest.

Open Access This article is distributed under the terms of the Creative Commons Attribution 4.0 International License (http://creativeco mmons.org/licenses/by/4.0/), which permits unrestricted use, distribution, and reproduction in any medium, provided you give appropriate credit to the original author(s) and the source, provide a link to the Creative Commons license, and indicate if changes were made.

\section{References}

1. Davis I, Milner CE, Hamill J (2004) Does increased loading during running lead to tibial stress fractures? A prospective study. Med Sci Sport Exerc 36:S58
2. Milner CE, Hamill J, Davis I (2007) Are knee mechanics during early stance related to tibial stress fracture in runners? Clin Biomech 22:697-703

3. Lafortune MA, Henning E, Valiant G (1995) Tibial shock measured with bone and skin mounted transducers. J Biomech 8:989-993

4. Laughton CA, Davis I, Hamill J (2003) Effect of strike pattern and orthotic intervention on tibial shock during running. J Appl Biomech 19:153-168

5. Barnes A, Wheat J, Milner CE (2011) Fore- and rearfoot kinematics in high- and low-arched individuals during running. Foot Ankle Int 32:710-171.6

6. Pearsall D, Henning E, Sterzing T (2002) The use of skin pretension to modify tibial bone acceleration estimates. World Congress of Biomechanics P1261, Calgary

7. Norris M, Anderson R, Kenny I (2014) Method analysis of accelerometers and gyroscopes in running gait: a systematic review. $\mathrm{J}$ Sport Eng Technol 228:3-15

8. Hamill J, Derrick T, Holt KG (1995) Shock attenuation and stride frequency during running. Hum Mov Sci 14:45-60

9. Fleiss JL (1986) The design and analysis of clinical experiments. Wiley, London

10. Lafortune MA, Lake MJ, Hennig E (1996) Differential shock transmission response of the human body to impact severity and lower limb posture. J Biomech 29:1531-1537

11. Crowell HP, Milner CE, Hamil J, Davis I (2010) Reducing impact loading during running with the use of real-time visual feedback. J Orthop Sports Phys Ther 40:206-213

12. Crowell HP, Davis I (2011) Gait retraining to reduce lower extrmeity loads in runners. Clin Biomech 26:78-83 